\title{
Editorial
}

\section{Comentario sobre la asociación entre la vía de parto y el daño perineal e incontinencia urinaria y fecal}

\author{
Drs. Anibal Faundes ${ }^{1}$, Laura Miranda ${ }^{1}$, Mauricio Cuello².
}

1 Centro de pesquisa em Saúde Reprodutiva de Campinas, Cemicamp.

Departamento de Ginecología y Obstetricia, Facultad de Ciencias Médicas, Universidade Estadual de Campinas, Sao Paulo, Brasil.

2 Division de Ginecología y Obstetricia, Escuela de Medicina, Pontificia Universidad Católica de Chile, Santiago, Chile.

\section{RESUMEN}

El parto vaginal se asocia con un riesgo más alto de daño perineal, incontinencia urinaria y fecal comparado con el parto cesárea. Dicho riesgo aumenta con el parto instrumental (ej. fórceps) y disminuye cuando la posición al momento del parto reduce la compresión sobre el sacro o si el parto ocurre bajo el agua. En otros factores que aumentan el riesgo de incontinencia se incluyen: la gran multiparidad, la duración del trabajo de parto, el peso fetal y tamaño de su cabeza, la edad muy joven al momento del primer parto, la inducción del trabajo de parto, la obesidad, la diabetes, la constipación, el tabaquismo y la historia de incontinencia urinaria o fecal. Factores sociales que se asocian a incontinencia incluyen la falta de educación, malas condiciones de hábitat, trabajo manual intenso, la falta de actividad física y el divorcio.

Palabras claves: incontinencia, parto vaginal, cesárea, parto instrumental, trauma.

\begin{abstract}
Vaginal delivery is associated with a higher risk of perineal injury, and urinary and fecal incontinence as compared with caesarean delivery; The risk is higher in case of operative vaginal delivery and lower if the position at delivery takes the weight off the sacrum or is carried out under water immersion; A number of other factors increase the risk of incontinence, including higher parity, duration of labour, fetal weight or size of fetal head, younger age at first delivery, induction of labour, obesity, diabetes, constipation, smoking and history of urinary or fecal incontinence. Social factors such as lack of education, poor living environment, intense manual labour, the absence of physical exercise and divorce, are also associated with incontinence.
\end{abstract}

Keywords: incontinence, vaginal delivery, caesarean delivery, instrumental delivery, injury.

\section{COMENTARIO}

Existen diferentes tipos de incontinencia urinaria entre los cuales se distinguen la incontinencia urinaria por esfuerzos, la incontinencia de urgencia miccional y la por rebalse. Con relación a la incontinencia fecal existen distintos grados de daño que se reflejan en la incapacidad de retener las heces, líquidos o gases.
Para este análisis de asociación entre la vía de parto con incontinencia urinaria y/o fecal incluimos cualquier forma de incontinencia tanto urinaria (IU) como fecal (IF). Esta revisión no se trata de una revisión sistemática dado que los artículos seleccionados no se limitan a los estudios randomizados de doble ciego sino que se incluyen todas las publicaciones identificadas en la materia. 
La revisión de la literatura deja pocas dudas sobre que la incidencia de incontinencia urinaria postparto es significativamente menor entre mujeres quienes tuvieron parto por cesárea comparado con aquellas con parto por vía vaginal (1-10). El riesgo de IU es más de dos veces mayor después de una parto vaginal comparado con el parto por cesárea. Sin embargo, parece que la incidencia, mas que la gravedad de la IU de esfuerzos, es la que se asocia con el tipo de parto (11). Por otro lado, tener un parto por cesárea no garantiza el evitar la IU puesto que el riesgo de ésta es mayor después de haber tenido un parto cesárea comparado con una nulípara (12).

El riesgo de IU es incluso mayor después de un parto instrumental comparado con un parto por cesárea $(2,4,13)$. Sin embargo, un estudio encontró que no había diferencia en la función del piso pélvico o sexual entre aquellas mujeres que tuvieron un parto instrumental sin complicaciones (exitoso) y aquellas que tuvieron parto por cesárea (14).

Mientras un estudio encontró que la asociación de parto vaginal con IU se limitaba a aquellos sin episiotomía (15) otros autores encontraron que el riesgo de IU aumentaba en los casos de laceración perineal o episiotomía $(16,17)$. Esta diferencia puede estar relacionada con la asociación entre características de la episiotomía y disfunción del piso pélvico. Un estudio mostró, por ejemplo, que las características de la episiotomía, particularmente un ángulo de 60 grados desde la línea media, podía ser protectora del daño del esfínter anal (18). Los mismos autores encontraron que la episiotomía con características protectoras no se asociaba con la incidencia de disfunción del piso pélvico (19). Una revisión sistemática respecto de las secuelas a largo plazo de la episiotomía concluyó que la evidencia disponible no apoya un rol de la episiotomía en la prevención de la IU, IF o del prolapso genital (20).

Un problema es que, tanto la episiotomía como las laceraciones graves del periné son indicadores de un parto dificultoso y, a menudo, ellos son confundidos por su asociación con parto vaginal instrumental, macrosomía fetal y una segunda fase prolongada del trabajo de parto $(21,22)$.

En general, la mayoría de los autores están de acuerdo con que los mismos factores asociados con IU e IF también se asocian con prolapso de los órganos pélvicos (POP), consecuentemente hay asociación entre POP e IU y entre IU e IF $(5,15,23)$.
Cuando se incluyen en el análisis sólo mujeres que han tenido un parto vaginal, la paridad $(5$, $16,24-30)$, la duración del trabajo de parto $(3,24,25$, 31 ), el peso fetal y el tamaño de la cabeza del feto se asocian significativamente con el riesgo de IU $(27,31-$ 33). En adición, el aumento de la edad (3, 6, 8, 10, 28, $29,34-38)$, edad más joven al primer parto $(3,27,32$, 33), la inducción del trabajo de parto (32) y las menstruaciones irregulares (8) también se asocian con un riesgo mayor de IU.

Otro factor que puede ser importante es la modalidad del parto, considerando que un estudio encontró que tener el trabajo de parto y el parto bajo el agua reducía el riesgo de IU (15).

La posición en el parto también parece ser un factor relacionado al riesgo de daño perineal. Un gran estudio que incluyó partos a domicilio en 4 países escandinavos separó la posiciones de parto en dos grupos: posiciones flexibles y no flexibles del sacro. Las posiciones que descomprimen el peso del sacro son: de rodillas, de pies, en cuatro pies, en cuclillas, de lado y sentada en la silla de parto. Posiciones definidas como no flexibles son semi-recostada, litotomía y supina. La prevalencia global de heridas reparadas fue $41,5 \%$. Las heridas perineales con necesidad de sutura se presentaron en el $60,9 \%$ de las mujeres primíparas y en el $36,8 \%$ de las multíparas. La prevalencia de episiotomía fue de $1 \%$. Las posiciones flexibles se asociaron con menos episiotomías después de ajustar por variables confundentes (primiparidad, peso nacimiento, traslado antes del parto y parto en el agua) (39).

El aumento del índice de masa corporal y la obesidad de la mujer han sido identificados como factores de riesgo para IU en prácticamente todos los estudios (2, 3, 6, 10, 26, 28, 33, 37, 38, 40-42). En concordancia con ello, también se ha encontrado una asociación entre IU e hiperlipidemia y consumo de alimentos ricos en grasas (37). En adición, un gran estudio realizado en Noruega encontró que la pérdida de peso postparto puede ser importante para evitar la incontinencia y recuperar la continencia en el postparto (43). Así también, se ha descrito un efecto benéfico de la cirugía bariátrica en reducir la IU (4446).

La diabetes $(5,6,24,28,37,47,48)$, una historia de infecciones del tracto urinario, incluyendo durante el embarazo $(3,15,24,33,49)$, la incontinencia durante el embarazo $(2,3)$, el aborto (29), la nicturia actual o en la infancia $(15,50)$, la tos 
crónica (5), la histerectomía (28), el uso de benzodiazepinas (51), la depresión (52), la constipación (47), y el tabaquismo $(28,38)$ son también identificados como factores de riesgo para incontinencia urinaria (29).

La asociación del tipo del parto con IF es, sin embargo, de algún modo controversial. Mientras algunos reportes describen una incidencia menor de IF en mujeres teniendo parto por cesárea $(6,16)$, una revisión sistemática y un meta-análisis encontraron que 'sólo 1 de 21 reportes demostraron una beneficio significativo del parto por cesárea en preservar la continencia anal; un reporte en el cual la incidencia de incontinencia fue extremadamente elevada, 39\% para el parto por cesárea y $48 \%$ para el parto vaginal, cuestionando, respecto a otros reportes, el momento y la naturaleza de la evaluación de la continencia. A mayor calidad del reporte, más cercana su Odds Ratio al 1' (53).

La laceración perineal, del esfínter y la episiotomía se asocian con un riesgo más alto de IF $(16,17)$. La gravedad de la laceración perineal, ya sea espontánea o por una episiotomía, durante el parto vaginal, parece constituir el factor más determinante de IF posterior al parto. Algunos autores encontraron que mujeres quienes sufrieron de lesiones obstétricas del esfínter anal reportan IF 4 a 5 veces más a menudo que las mujeres sin tales lesiones (19).

La constipación, la tos crónica y la hipertensión han sido asociadas significativamente con IF $(17,26,31)$ como también la edad, la obesidad (6), y la diabetes $(5,26)$.

Sin embargo, algunas de estas asociaciones son inconsistentes y con estudios diferentes llegando a conclusiones opuestas. Por ejemplo, un estudio encontró que el parto vaginal conllevaba cambios del tipo prolapso e IU objetiva pero sin un aumento en el auto-reporte de disfunción del piso pélvico (IU o IF) a los 6 meses postparto comparado con mujeres quienes tuvieron parto por cesárea sin segunda fase de trabajo de parto (54). En otros estudios, el peso del recién nacido más grande y la paridad no fueron asociados con el riesgo de IU (2). Otro autor no encontró asociación de la IU con la edad al primer y último nacimiento, la episiotomía, el peso de nacimiento sobre 4 kgs., y el tabaquismo (24).

Varios factores sociales han sido también asociados con IU, entre ellos la falta de educación, malas condiciones de hábitat (específicamente vivir en áreas rurales), trabajo manual intenso, la falta de actividad física y el divorcio o la viudez $(29,37)$. De manera similar, la prevalencia de IF en mujeres nulíparas se encontró estar asociada con bajo nivel educacional además de a co-morbilidades (16, 29).

La incontinencia urinaria y fecal no son fatales, pero deterioran la calidad de vida (CdV), lo cual puede ser de manera severa. Un estudio encontró relaciones significativas entre los escores de CdV y la frecuencia de IU $(p=0,002)$ y la cantidad de pérdida de orina $(p=0,002)$. El impacto en los escores de CdV osciló desde nivel leve, moderado a severo (29). Otro estudio encontró que mujeres con IF tuvieron peores puntajes en el self-rated health utility index score y en el Medical Outcomes Study Short Form Health Survey (SF-12) mental summary score que mujeres sin incontinencia. De manera similar, mujeres con IU tuvieron peores puntajes en ambos tests comparado con aquellas sin incontinencia. Las mujeres con IU e IF tuvieron los puntajes más bajos en el test SF-12 $(55,56)$.

\section{Conclusiones}

Aunque existen otros factores asociados con un mayor o menor riesgo de incontinencia urinaria y fecal, el riesgo de ambas incontinencias es mayor después de un parto vaginal comparado con un parto por cesárea. El parto vaginal instrumental, el cual puede causar daño del esfínter, se asocia con el mayor riesgo de IF, mientras el parto vaginal en posición flexible como es la posición vertical o la modalidad de parto bajo el agua reducen el riesgo de trauma perineal y por ende el de incontinencia.

\section{REFERENCIAS}

1. MacLennan AH, Taylor AW, Wilson $\mathrm{DH}$, Wilson $\mathrm{D}$. The prevalence of pelvic floor disorders and their relationship to gender, age, parity and mode of delivery. Int J Gynecol Obstet. 2000 Dec; 107(12):1460-70

2. Eason E, Labrecque M, Marcoux S, Mondor M. Effects of carrying a pregnancy and of method of delivery on urinary incontinence a prospective cohort study. BMC Pregnancy Childbirth. 2004; 4: 4

3. Fritel X, Fauconnier A, Levet C, Bénifla J-L. Stress urinary incontinence 4 years after the first delivery: 
a retrospective cohort survey. Acta Obstet Gynecol Scand. 2004 Oct; 83(10): 941-945

4. Handa VL, Blomquist JL, Knoepp LR, Hoskey KA, McDermott KC, Muñoz A. Pelvic floor disorders 510 years after vaginal or cesarean childbirth. Obstet Gynecol 2011 Oct; 118(4):777-84

5. Perera J, Kirthinanda DS, Wijeratne S, Wickramarachchi TK. Descriptive cross sectional study on prevalence, perceptions, predisposing factors and health seeking behavior of women with stress urinary incontinence. BMC WomenHealth. 2014; $14: 78$

6. Hallock JL, Handa VL. The epidemiology of pelvic floor disorders and childbirth: an update. Obstet Gynecol Clin North Am. 2016 Mar; 43(1): 1-13

7. Teunissen TA, van den Bosch WJ, van den Hoogen HJ, Lagro-Janssen AL. Prevalence of urinary, fecal and double incontinence in the elderly living at home.Int Urogynecol J Pelvic Floor Dysfunct. 2004 Jan-Feb; 15(1):10-3

8. Komeilifar R, Javadifar N, Afshari P, Haghighizade $\mathrm{MH}$, Honarmandpour A. The Prevalence, Subtypes and Obstetric Risk Factors of Urinary Incontinence in Reproductive Age Women Referred to Community Health Care. Iran,; Int J Community Based Nurs Midwifery. 2017 Jul; 5(3): 275-283

9. Keag OE, Norman JE, Stock SJ. Long-term risks and benefits associated with cesarean delivery for mother, baby, and subsequent pregnancies: Systematic review and meta-analysisPLoS Med. 2018 Jan; 15(1): e1002494

10. Qi X, Shan J, Peng L, Zhang C, Xu F. The effect of a comprehensive care and rehabilitation program on enhancing pelvic floor muscle functions and preventing postpartum stress urinary incontinence. Medicine (Baltimore) 2019 Aug; 98(35): e16907

11. Hutton EK, Hannah ME, Willan AR, Ross S, Allen AC, Armson BA, Gafni A, Joseph KS, Mangoff K, Ohlsson A, Sanchez JJ, Asztalos EV, Barrett JFR, the Twin Birth Study Collaborative Group. Urinary incontinence and other maternal outcomes 2 years after caesarean or vaginal birth for twin pregnancy: a multicentre randomised trial. BJOG. 2018 Dec; 125(13): 1682-1690

12. Faundes A, Guarisi T, Pinto-Neto AM. The risk of urinary incontinence of parous women who delivered only by cesarean section. Int J Gynecol Obstet. 2001 72: 41-46

13. Blomquist JL, Muñoz A, Carroll M, Handa VL. Association of Delivery Mode With Pelvic Floor Disorders After Childbirth. JAMA. 2018 Dec 18; 320(23): 2438-2447

14. Crane Ak, Geller EJ, Bane H, Rujin Ju, Myers E,. Matthews CA. Evaluation of Pelvic Floor Symptoms and Sexual Function in Primiparous Women Who Underwent Operative Vaginal Delivery Versus Cesarean Delivery for SecondStage Arrest; Female Pelvic Med Reconstr Surg. 2013 Jan-Feb; 19(1): 13-16

15. Liu Y, Liu B, Huang X, Du C, Peng J, Huang P, Zhang J. A comparison of maternal and neonatal outcomes between water immersion during labor and conventional labor and delivery. BMC Pregnancy Childbirth. 2014; 14: 160

16. Laine K, Skjeldestad FE, Sandvik L, Staff AC. Prevalence and Risk Indicators for Anal Incontinence among Pregnant Women. ISRN Obstet Gynecol. 2013; 2013: 947572

17. Subki $A H$, Fakeeh MM, Hindi MM, Nasr AM, Almaymuni AD, Abduljabbar HS. Fecal and Urinary Incontinence Associated with Pregnancy and Childbirth. Mater Sociomed. 2019 Sep; 31(3): 202-206

18. Stedenfeldt M, Pirhonen J, Blix E, Wilsgaard T, Vonen B, Øian P. Episiotomy characteristics and risks for obstetric anal sphincter injuries: a casecontrol study. BJOG. 2012 May; 119(6):724-30

19. Stedenfeldt M, Pirhonen J, Blix E, T Wilsgaard T, Vonen B, Øian P. Anal incontinence urinary incontinence and sexual problems in primiparous women: a comparison between women with episiotomy only and women with episiotomy and 
anal sphincter injury. BMC Womens Health. 2014; 14: 157

20. Hartmann K, Viswanathan M, Palmieri R, Gartlehner G, Thorp J, Jr, Lohr KN. Outcomes of routine episiotomy: a systematic review. JAMA. 2005;293(17):2141-2148

21. Kearney R, Miller JM, Ashton-Miller JA, Delancey JO. Obstetric factors associated with levator ani muscle injury after vaginal birth. Obstet. Gynecol. 2006;107:144-149

22. Memon HU, Handa VL. Vaginal childbirth and pelvic floor disorders. Women Health (Lond Engl). 2013 May; 9(3): 10.2217

23. Samuelsson E, Victor A, Svärdsudd K. Determinants of urinary incontinence in a population of young and middle-aged women. Acta Obstet Gynecol Scand. 2000 Mar; 79(3):20815

24. Kılıç $M$. Incidence and risk factors of urinary incontinence in women visiting Family Health Centers. Springerplus. 2016; 5(1): 1331

25. Masenga GG, Shayo BC, Msuya S, Rasch V. Urinary incontinence and its relation to delivery circumstances: A population-based study from rural Kilimanjaro, Tanzania. PLoS One. 2019; 14(1): e0208733

26. Rortveit G, Subak LL, Thom DH, Creasman JM, Vittinghof EF,. Van Den Eeden SK, Brown JS. Urinary incontinence and pelvic organ prolapse in a population- based, racially diverse cohort: prevalence and risk factors. Female Pelvic Med Reconstr Surg. 2010 Sep; 16(5): 278-283

27. Seshan V, Muliira JK. Self-reported urinary incontinence and factors associated with symptom severity in community dwelling adult women: implications for women's health promotion. BMC Women Health. 2013; 13: 16

28. Danfort H, Townsend MH, Lifford K, Curhan GC, Resnick NM, Grodstein F. Risk Factors for Urinary Incontinence among Middle-aged Women. : Am J Obstet Gynecol. 2006 Feb; 194(2): 339-345.
29. Demircan N, Özmen U, Köktürk F, Küçük H, Ata S, Harma M, Arıkan II. What are the probable predictors for vurinary incontinence during pregnancy?. PeerJ. 2016; 4: e2283

30. Zhou H-H, Shu B, Liu T-Z, Wang X.H, Yang Z.H, Guo Y-L. Association between parity and the risk for urinary incontinence in women: A metaanalysis of case control and cohort studies. Medicine (Baltimore) 2018 Jul; 97(28): e11443

31. Ajith AK. Prevalence and Factors of Urinary Incontinence among Postmenopausal Women Attending the Obstetrics and Gynecology Outpatient Service in a Tertiary Health Care Center in Kochi, Kerala. Indian J Community Med. 2019 Oct; 44(Suppl 1): S30-S33

32. Thom DH, Brown JS, Schembri M, Ragins AI, Creasman JM, Van Den Eeden SK. Neurourol Urodyn. 2011 Nov; 30(8): 1456-1461

33. Pizzoferrato A-C, Fauconnier A, Quiboeuf E, Morel K, Schaal JP, Fritel X. Urinary incontinence after first delivery: Risk factors associated with prevalence, incidence, remission, and persistence in a cohort of 236 women. Neurourol Urodyn. 2014 Nov; 181: 259-266

34. Nygaard I, Barber MD, Burgio KL, Kenton K, Meikle S, Schaffer J, Spino C, Whitehead WE, Wu J, Brody DJ. Pelvic Floor Disorders Network.Prevalence of symptomatic pelvic floor disorders in US women. JAMA. 2008 Sep 17; 300(11):1311-6

35. Teunissen D, , van Weel C, Lagro-Janssen T. Urinary incontinence in older people living in the community: examining help-seeking behaviour. $\mathrm{Br}$ J Gen Pract. 2005 Oct 1; 55(519): 776-782

36. Progetto Menopausa Italia Study Group Risk factors for genital prolapse in nonhysterectomized women around menopause. Results from a large cross-sectional study in menopausal clinics in Italy. Eur J Obstet Gynecol Reprod Biol. 2000 Dec; 93(2):135-40 
37. Liu B, Wang L, Huang S-S, Wu Q, Wu D-L. Prevalence and risk factors of urinary incontinence among Chinese women in Shanghai. Int J Clin Exp Med. 2014; 7(3): 686-696

38. Bump RC, Norton PA. Epidemiology and natural history of pelvic floor dysfunction. Obstet Gynecol Clin North Am. 1998;25:723-746

39. Edqvist M, Blix E, Hegaard HK, Ólafsdottir OA, Hildingsson I, Ingversen K, Mollberg M, Lindgren $\mathrm{H}$. Perineal injuries and birth positions among 2992 women with a low risk pregnancy who opted for a homebirth.BMC Pregnancy Childbirth. 2016; 16: 196

40. Fornell E U, Wingren G, Kjølhede P. Factors associated with pelvic floor dysfunction with emphasis on urinary and fecal incontinence and genital prolapse: an epidemiological study. Acta Obstet Gynecol Scand. 2004 Apr; 83(4):383-9

41. Melville JL, Katon W, Delaney K, Newton K. Urinary incontinence in US women: a populationbased study. Arch Intern Med. 2005 Mar 14; 165(5):537-42

42. Pomian A, Lisik W, Kosieradzki M, Barcz E. Obesity and Pelvic Floor Disorders: A Review of the Literature. Med Sci Monit. 2016; 22: 18801886

43. Wesnes SL, Hunskaar S, Bo K, Rortveit G. Urinary inciontinence and weight change during pregnancy and post partum, A chort study..Am J Epidemiol. 2010 Nov 1; 172(9): 1034-1044

44. Subak LL, King WC, Belle SH, PhD, Chen J-Y, Courcoulas, AP, . Ebel FE, Flum DR, Khandelwal S, Pender JR, Pierson SK, Pories WJ, Steffen KJ, Strain GW, Wolfe BM, Huang AJ. Urinary Incontinence Before and After Bariatric Surgery. JAMA Intern Med. 2015 Aug 1; 175(8): 1378-1387

45. Bulbuller N, Habibi M, Yuksel M, Ozener O, Oruc MT, Oner OZ, Kazak MA. Effects of bariatric surgery on urinary incontinence. Ther Clin Risk Manag. 2017; 13: 95-100
46. Yuksel M, Ozener O, Mehmet Tahir Oruc MT, Oner OZ, Kazak MA. Effects of bariatric surgery on urinary incontinence. Bulbuller N, Habibi M. Ther Clin Risk Manag. 2017; 13: 95-100

47. Sensoy N, Dogan N, Ozek B, Karaaslan L. Urinary incontinence in women: prevalence rates, risk factors and impact on quality of life. Pak J Med Sci. 2013 May-Jun; 29(3): 818-822

48. Aruna V. Sarma AV, Kanaya A, Nyberg LM, Kusek JW, Vittinghoff E, Rutledge B, Cleary PA, Gatcomb P, Brown JS. Diabetes Control and Complications Trial/Epidemiology of Diabetes Interventions and Complications Research Group (DCCT/EDIC). Risk Factors for Urinary Incontinence Among Women with Type 1 Diabetes: Findings from the Epidemiology of Diabetes Interventions and Complications Study. Urology. 2009 Jun; 73(6): 1203-1209

49. Moore EE, Jackson SL, Boyko EJ, Scholes D, Finn SD. Urinary incontinence and urinary tract infection: temporal relationships in postmenopausal women. Obstet Gynecol. 2008 Feb; 111(2 Pt 1):317-23

50. Fitzgerald MP, Thom DH, Wassel-Fyr C, Subak L, Brubaker L, Van Den Eeden SK, Brown JS. Childhood urinary symptoms predict adult overactive bladder symptoms. Reproductive Risks for Incontinence Study at Kaiser Research Group. J Urol. 2006 Mar; 175(3 Pt 1):989-93

51. Landi F, Cesari M, Russo A, Onder G, Sgadari A, Bernabei R, Silvernet-HC. Benzodiazepines and the risk of urinary incontinence in frail older persons living in the community. Clin Pharmacol Ther. 2002 Dec; 72(6):729-34

52. Bortolotti A, Bernardini B, Colli E, Di Benedetto P, Giocoli Nacci G, Landoni M, Lavezzari M, Pagliarulo A, Salvatore S, von Heland M, Parazzini F, Artibani W. Prevalence and risk factors for urinary incontinence in Italy. Eur Urol. 2000 Jan; $37(1): 30-5$

53. Nelson RL, Furner SE, Westercamp M, Farquhar C. Cesarean delivery for the prevention of anal incontinence. Cochrane Database Syst Rev. 
2010, Feb 17;(2):CD006756. doi: 10.1002/14651858.CD006756.pub2

54. Rogers RG, Leeman LM, Borders N, Qualls C, Fullilove AM, Teaf D, Hall J, Bedrick E, Albers LL. The contribution of the second stage of labor to pelvic floor dysfunction: A prospective cohort comparison of nulliparous women . BJOG. 2014 Aug; 121(9): 1145-1154

55. Handa VL, Zyczynski HM, Burgio KL, Fitzgerald MP, Borello-France D, Janz NK, Fine PM,
Whitehead W, Brown MB, Weber AM. The impact of fecal and urinary incontinence on quality of life 6 months after childbirth. Am J Obstet Gynecol. 2007 Dec; 197(6): 636.e1-636.e6

56. Fialkow MF, Melville JL, Lentz GM, Miller EA, Miller J, Fenner DE. The functional and psychosocial impact of fecal incontinence on women with urinary incontinence. Am J Obstet Gynecol. 2003 Jul;189(1):127-9 